\title{
A Comparative Performance Analysis between Saturated Core Voltage Regulators Using Silicon Steel and Amorphous Alloys
}

\author{
C. S. Chaves ${ }^{1}$, J. C. Oliveira ${ }^{2}$ and J. A. F. Barbosa Jr. ${ }^{2}$ \\ ${ }^{1}$ Department of Electronic - Instituto Federal do Triângulo Mineiro (IFTM) \\ Líria Terezinha Lassi Capuano Av., 255, 38740-000 Patrocínio, Minas Gerais (Brazil) \\ Phone:+55 349149 4344, e-mail: camillachaves@iftm.edu.br \\ ${ }^{2} \mathrm{NQEE}$ - Universidade Federal de Uberlândia (UFU) \\ João Naves de Ávila Av., 2121, 38408-100 Uberlândia, Minas Gerais (Brazil) \\ e-mail: jcoliveira@ufu.br, johnareis@gmail.com
}

\begin{abstract}
The focus of the paper presented herein is directed towards the investigative study of the operational performance of saturated reactors intended for voltage regulation, which consist of magnetic silicon steel cores and amorphous alloys. In order to reach the defined goal, two similar devices in terms of voltage operation and reactive power compensation are employed, both embodied with a twin-tripler or 12 pulse configuration. Both devices are modelled and implemented onto the ATP simulator to carry out comparative studies. Through the use of a classic distribution feeder, performance studies concerning sudden voltage variations are considered, which are focused on above all: evaluation of active losses, non-saturated and saturated reactive power, waveforms for inductive current and voltage with their harmonic spectra. The results obtained from the studies allows for a prompt correlation between the characteristics associated with the functionality of the device, highlighting the advantages offered by the use of one or other magnetic material.
\end{abstract}

\section{Keywords}

Magnetic alloys, power quality, reactive power, saturated reactor core voltage regulation

\section{Introduction}

Many applications today require the use of very long transmission lines, aimed at interlinking diverse geographical regions involved in the formation of integrated systems. In such settings, relief loads can bring significant impacts to the voltage levels. In a similar manner, other situations involving distribution radial feeders with load variations of significant size may equally result in voltage alterations. In fact, countless situations imply voltage levels that violate the limits approved by law [1]. Given this situation, the solutions used are usually presented in the form of the installation of voltage regulation devices. These devices, in line with their operating principles, are based on two basic concepts:

- Direct compensation - modification of tapes or voltage compensation injection;
- Indirect compensation [2] - control of reactive power by devices, such as capacitor banks and reactors (which are controlled or not), synchronized compensators and others founded on electronic technologies [3]; classic saturated core reactors, for which the advantages and disadvantages are amply discussed in [2], [4].

It should be highlighted here that the composition of this saturated reactor with its capacitor bank results in the socalled Static Compensator Saturated Reactive Core (SCSRC). This type of equipment was widespread during the 1960 `s, and which is still the objective of present day investigative work. Examples of such developments can be found in [4]-[6]. The intrinsic performance of this device offers dynamic reactive power consumption (reactor) and supply (capacitor) properties, as well as affords the product the function of regulating voltage elevations and reductions.

Without going into the discriminations of the above solution, when it comes to some specific applications, the use of saturated core regulator technology still presents itself as an option to be considered. This is due to its robustness, economy of initial investments and maintenance requirements, good efficiency in voltage regulation and rapid response.

With the focus on the inductive component of the device, it is important to highlight that the operational philosophy of the compensator is founded upon its voltage and current (VxI) feature. This, as already known, is determined by the magnetic material employed in the core, which implies that under low level voltages or steady-state conditions (as frequently occurs under full load), the reactor operates in a region below saturation. Thus, it demands a small reactive power from the system, (this should ideally be null). As the line load is reduced, the voltage may increase and under these circumstances, the device is brought to a higher saturation state. This is a crucial fact for the determination of the higher 
consumption of reactive power, thus resulting in effects associated with voltage reduction.

Through the combination of such features as the magnetic core with windings and special interconnections, the developments made in [4], [5], by using as a premise the 12 pulse configuration or twin-tripler, resulted in prototypes with promising performance in computational simulations as well as experimental tests. However, the search for properties such as reduction of losses, lower reactive currents under steady-state conditions and improvements to harmonic distortions, still present themselves as research motivating themes.

In light of the above, the possibility arises of constructive ideas with new materials, like amorphous alloys. In fact, various documents and research focused on the building of transformers with such cores indicate expressive efficiency improvements to the equipment, such as the reduction in iron loss [7]. This results in important perspectives to be considered, and within this scenario, the current study presents its principal objective, which is to correlate operational aspects associated with two devices, one made up of a reactor with a silicon steel core while the other with an amorphous alloy core. To this end, this study is based on computational models, which have already been developed [4], [8] and implemented on the ATP platform.

As the study is grounded in these recourses, it contemplates the results of investigations related to the efficiency of the regulation process, the values of the current demanded by the regulators, the waveforms and frequency spectrums, besides active losses on the windings and core. The computational results are therefore presented and discussed to establish a comparative basis between the two technologies, concerning the type of material used for the composition of the magnetic cores of the two regulators.

\section{Silicon Steel and Amorphous Alloys Properties}

Amorphous alloys are known to provide a significant decrease in hysteresis loss and thus core loss. To illustrate these differences, the upper halves of the hysteresis loop, given by the $\mathrm{BxH}$ relationship for the silicon steel $\mathrm{AK}$ Steel Oriented M-6 CARLITE $^{\circledR} 0.35 \mathrm{~mm}$ and the amorphous alloys Metglas ${ }^{\circledR}$ 2605SA1, are compared in Figure 1. In Figure 2, information concerning the core loss is given, in relation to induction $\mathrm{B}$, where it is possible in this sense to compare the silicon steel to the amorphous alloy.

Given the properties clearly synthesized in the previous figures, there arises the consequence of the advantages associated to the use of amorphous alloys in substitution of the conventional silicon steel for the building of voltage regulators considered herein. The smaller current value under the system`s nominal operational conditions, implies in a non-saturated state of the magnetic material resulting in lower Joule loss, and a drastic decrease in iron loss, denoting greater efficiency of the equipment.

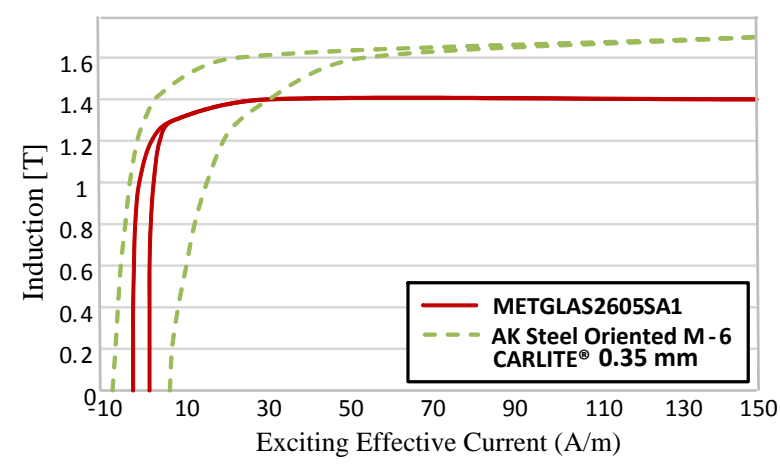

Fig. 1. Comparison between the hysteresis loops for the amorphous alloys and silicon steel.

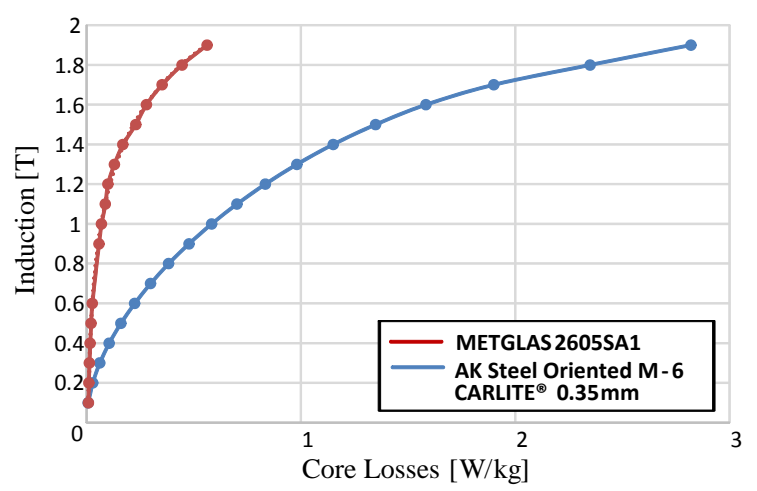

Fig. 2. Comparative values of the core losses for silicon steel and amorphous alloys.

\section{Physical Constitution and Modelling of the Twin-Tripler Saturated Reactors on the ATP Simulator}

In [4], [8] the physical arrangement of a saturated reactor and its respective modelling on the ATP simulator are presented in detail. As one can see, the device in question shows similar operational principles to a saturated transformer, still emphasising, however, that the product under focus is presented as being made up of two identical three-phase arrangements, electrically interconnected, where each one is established as having three magnetic columns, as shown in the topology illustrated in Figure 3.

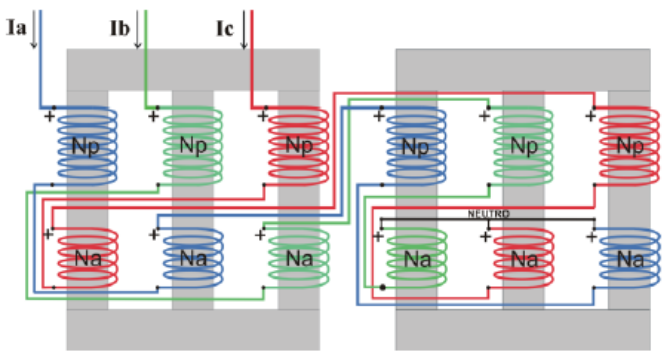

Fig. 3. Saturated Core Reactor - constructive twin-tripler or 12 pulse arrangement.

In each of the magnetic columns one finds two windings, one is the main winding the other auxiliary. These are electrically and magnetically interlinked between themselves as well as with the coils from the neighbouring columns. 
In regards to the modelling and implementation of the device on the ATP simulator consistent with [4], [5], [8], the final product can be found synthesized in Figure 4.

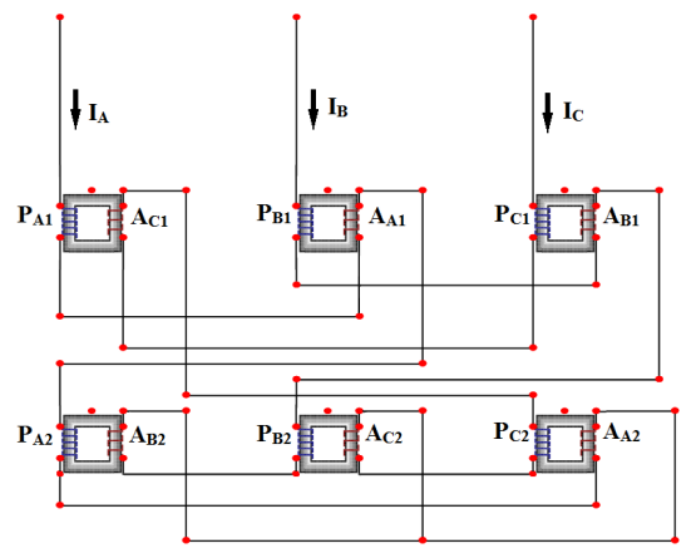

Fig. 4. The twin-tripler arrangement on the ATP Simulator.

As indicated, each element corresponds to a single-phase magnetic core, and the six structures have identical magnetized curves. The single-phase units have a principal coil (P) and an auxiliary coil (A). The point situated above each element allows access to the magnetizing branch of the equivalent electrical circuit of the component, in order to be able to implement hysteresis loops and additional features from the core. However, for the purposes of the studies conducted in this paper, only the nonlinearity magnetization curves were used under the terms shown in Figure 5, therefore, the hysteresis loop was not considered.

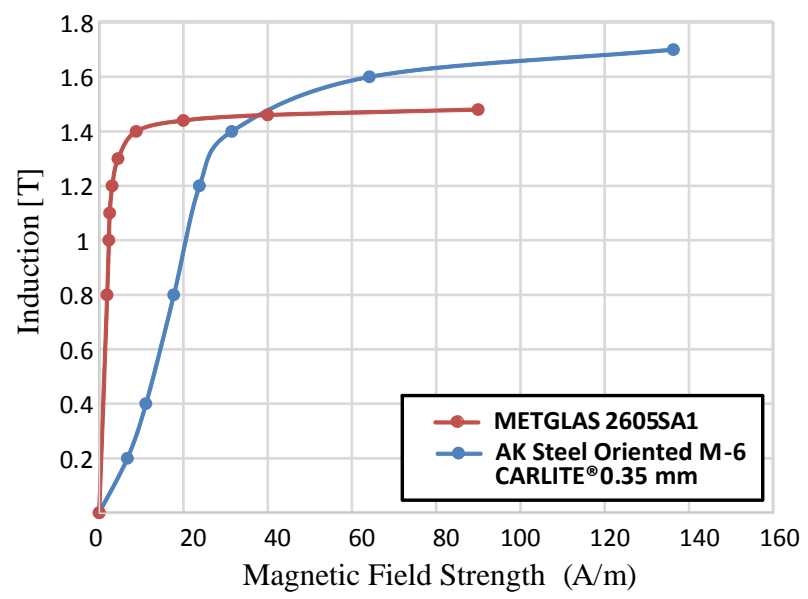

Fig. 5. Amorphous Alloys magnetized curves METGLAS 2605SA1 and for the silicon steel AK Steel Oriented M-6.

As indicated, the saturation knee curve referring to silicon steel corresponds to a magnetic induction value higher than that for the amorphous alloy. This means that under non-saturated conditions for both materials, the core made up of amorphous alloys will have a lower current demand than that of silicon steel one.

By use of the procedures detailed in [8], it is possible to correlate the $\mathrm{BxH}$ curve with the respective feature $\lambda \mathrm{xI}$ linked to a given reactor, where $\lambda$ is the concatenated flux and I the peak current consumed by the reactor.

\section{Characterization of the System for the Case Studies}

With the intention of evaluating the operational performance of both regulators, the simplified radial electrical system indicated in Figure 6 was adopted for the realization of the studies. This corresponds to a typical distribution feeder. As indicated, the focus of the studies is directed towards the $34.5 \mathrm{kV}$ busbar, where one finds the load installed that will be the object of abrupt changes and respective impacts over the voltage. Among the main characteristics of the system used, the following are highlighted: the level of short circuit on the $138 \mathrm{kV}$ bus is $200 \mathrm{MVA}$; the transformers T1 and T2 are 10 MVA, with an impedance percentage of $10 \%$; the line is represented by an equivalent circuit $\pi$ with a resistance of $0.10 \Omega / \mathrm{km}$, inductance of $0.60 \mathrm{mH} / \mathrm{km}$ and capacitance of $4.85 \eta \mathrm{F} / \mathrm{km}$ and finally, the supplied load is of $5 \mathrm{MVA}$ under normal operating conditions.

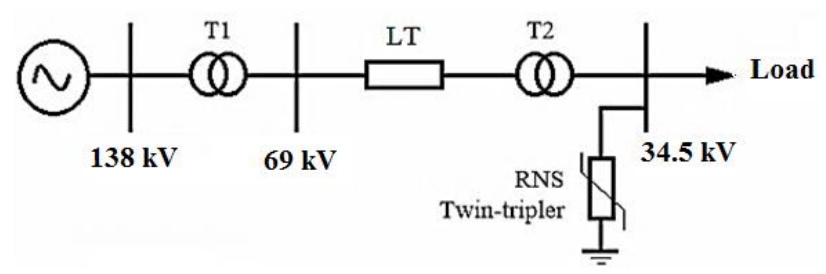

Fig. 6. Electrical radial feeder used for the computational studies.

From a hypothetical working scenario, associated with a sudden $80 \%$ load loss, it was proceeded the basic constructive parameters related to the two saturated reactors (silicon steel and amorphous alloys) for setting the terminal voltage to the desired standards. Some information of reference can be verified on Table I. The data refers to the physical magnetic core dimensions and number of turns to the reactor windings. These figures were obtained from the procedures described in [9]. Besides this, the curves $\lambda x I$ were considered in accordance with the features of each magnetic material.

Table I. - Constructive Features of the Saturated Reactors

\begin{tabular}{|c|c|c|}
\hline CONSTRUCTIVE FEATURES & $\begin{array}{c}\text { SILICON } \\
\text { STEEL }\end{array}$ & $\begin{array}{c}\text { AMORPHOUS } \\
\text { ALLOYS }\end{array}$ \\
\hline Nominal Power [kVAr] & 1500 & 1500 \\
\hline Saturation Voltage [kV] & 35.9 & 35.9 \\
\hline Frequency [Hz] & 60 & 60 \\
\hline $\begin{array}{c}\text { Number of Turns } \\
\text { (Main Winding) }\end{array}$ & 475 & 475 \\
\hline $\begin{array}{c}\text { Number of Turns } \\
\text { (Auxiliary Winding) }\end{array}$ & 173 & 173 \\
\hline $\begin{array}{c}\text { Magnetic Section of the Core } \\
{\left[\mathrm{cm}^{2}\right]}\end{array}$ & 372.78 & 454.32 \\
\hline Window Height [mm] & 200 & 250 \\
\hline Column Width [mm] & 136 & 171 \\
\hline Window Length [mm] & 1790 & 1620 \\
\hline
\end{tabular}

For the investigative studies, three operational situations were used. They are:

- Case 1 - system with normal load and occurrence of a sudden relief of load, characterized 
by a reduction of $80 \%$ of the initial value of $5 \mathrm{MVA}$ in $\mathrm{t}=1 \mathrm{~s}$, with no voltage regulation device;

- Case 2 - as above, but considering the action of the twin-tripler saturated core regulator, made up of silicon steel;

- Case 3 - as above, but substituting the magnetic material of the reactor for amorphous alloys.

\section{Computational Performance Studies}

In line with the characteristics imposed on the system, reactors and operational conditions, the following presents the results for the computational studies carried out from the already considered distribution feeder and operational conditions.

\section{A. Results for Case 1 - System with no Voltage Regulator}

With the view to establishing a basis for the comparison of system performance during the specified sudden load variation, one has the voltage profile indicated in Figure 7. This, as already informed, corresponds to the results obtained for the $34.5 \mathrm{kV}$ bus.

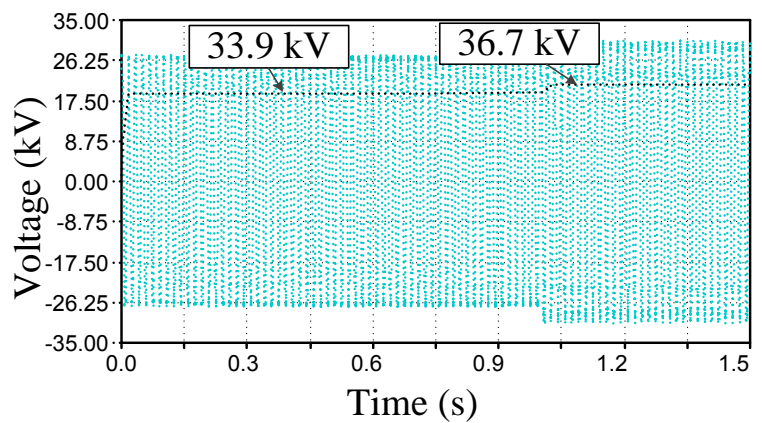

Fig. 7. Voltages for the $34.5 \mathrm{kV}$ bus- before and after the $80 \%$ load relief occurred to the 5 MVA load.

The results show that for the initial situation, the bus voltage is presented at around $0.98 \mathrm{pu}$, and after the load relief this magnitude reached a value of $1.064 \mathrm{pu}$. With the base voltage equal to $34.5 \mathrm{kV}$, this means that under the condition imposed by the load relief, the system exceeds the admissible limits [1], therefore making it necessary to proceed towards the restoration of the said limits to a standard considered as acceptable. As highlighted, this was carried out through the insertion of a voltage regulator, consisting of one or other saturated reactor, with those properties already explored in the text.

\section{B. Results for Case 2 - System with the Silicon Steel Saturated Reactor}

The study was then repeated but this time under the condition of a saturated reactor built with magnetic materials based on silicon steel, which possess the constructive features indicated in Table I. Under these conditions, the performances obtained are shown in Figure 8.

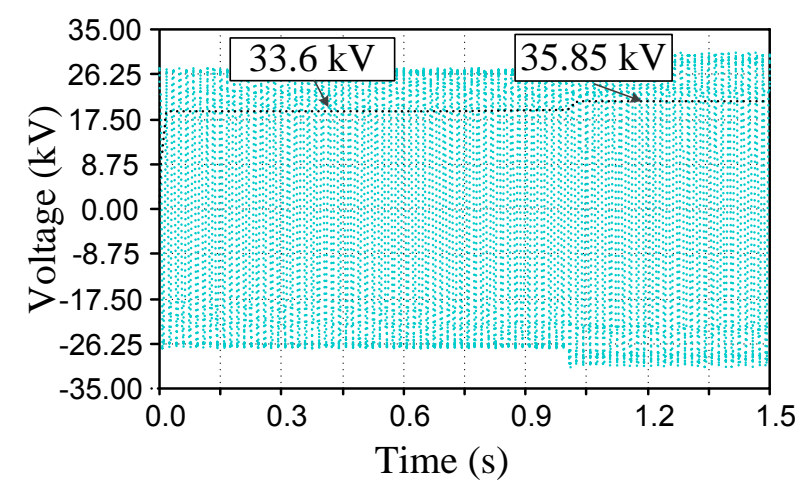

Fig. 8. Voltages for the $34.5 \mathrm{kV}$ bus with the silicon steel Twin-Tripler Reactor - before and after load relief.

One notes that, the load loss resulted in an instantaneous voltage variation. This indicates that in the prior load loss condition as well as in the saturated reactor scenario, the voltage, which before was around $0.97 \mathrm{pu}$, after the load rejection, increases to a level around the 1.04 pu mark. Therefore, the silicon steel regulator device provides an adequate voltage level at its limit value. It must be emphasised that the degree of compensation is strictly linked to the power of the reactor used. Therefore, other devices with a higher power level could produce a better voltage regulation result, however, this was not taken into account in the paper.

When it comes to the current absorbed by the saturated reactor, its behaviour along the study period is shown in Figure 9.

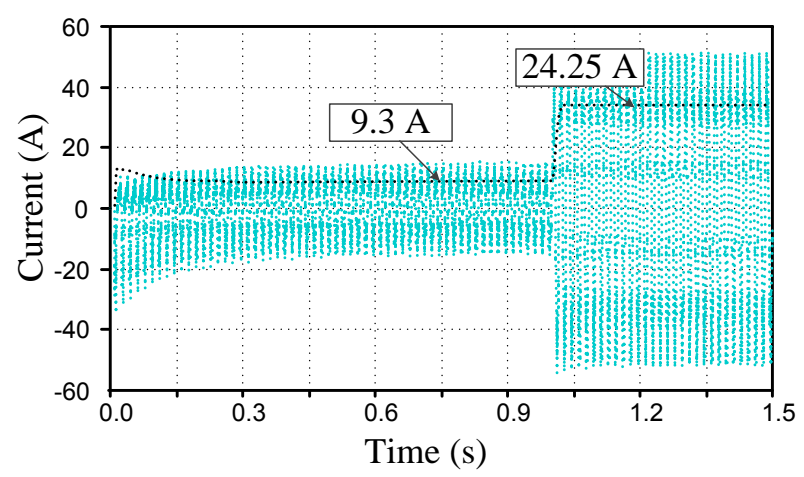

Fig. 9. Currents absorbed by the silicon steel reactor - before and after the load relief.

Examined in Figure 9 is the performance of the reactor on the imposed operating conditions, which consisted of the start-up, steady-state and load rejection periods. It is worth noting that the initial reactor current is $9.3 \mathrm{~A}$ and after the load relief, this magnitude went up to $24.25 \mathrm{~A}$. This value is practically equal to nominal reactor current under saturation.

This current, in accordance with [2], is presented in nonsinusoidal waveform, and as is already known produces expected characteristic harmonic order components. Of these, for the full reactor operation scenario (when there is load rejection), one notes that besides the fundamental component, there arise, concomitantly, the dominant harmonic orders: $\mathrm{I}_{11}=3.37 \mathrm{~A}$ e $\mathrm{I}_{23}=1.07 \mathrm{~A}$. These as known are injected onto the supply network and result in 
voltage harmonic distortions, which are presented as waveforms illustrated in Figures 10 (a) and (b). By carrying out a spectral analysis of the voltages on the $34.5 \mathrm{kV}$ busbar, it can be noted that the voltage waveform, under normal load conditions, is shown to have a THD of $3.89 \%\left(\mathrm{~V}_{11}=3.67 \%\right.$ or $760 \mathrm{~V}$ and $\mathrm{V}_{23}=0.28 \%$ or $59 \mathrm{~V}$, phase values).

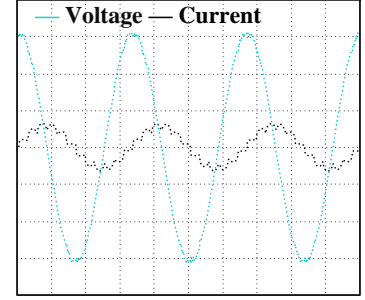

(a)

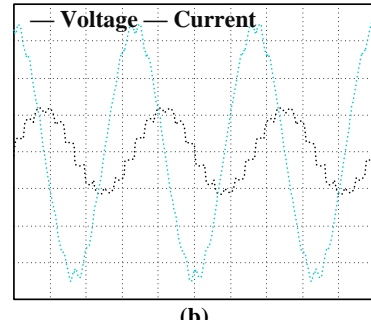

(b)
Fig. 10. Details for the voltage waveforms for the operational conditions of the silicon steel reactor: (a) before the load relief and (b) after the load reduction.

Another relevant magnitude to the process studied herein, refers to the magnetic flux density that the saturated reactor experiences during its operation. In the load prerejection condition, this is around $1.83 \mathrm{~T}$, and after rejection, a level of $1.95 \mathrm{~T}$ is reached. This finding is directly associated with the correlation between the flux and the peak current of the reactor. As a consequence, the silicon steel reactor, in the lowest and full saturation condition and in light of the values presented in Figure 2, one obtains the corresponding active losses, which are shown, respectively, to be of the order $2.49 \mathrm{~W} / \mathrm{kg}$ and $3.05 \mathrm{~W} / \mathrm{kg}$.

\section{Results for Case 3 - System with the Amorphous Alloy Saturated Reactor}

Maintaining the same logical path as previously used, Figure 11 shows the profile of the instantaneous voltages and their corresponding efficient values during the study period. These show that prior to the load reduction, the supply voltage is approximately $0.98 \mathrm{pu}$ and after load rejection, around $1.04 \mathrm{pu}$. Under these terms, the amorphous alloy regulating device indicates, in respect to the regulating process, a similar performance to that of the silicon steel. The same previous consideration concerning the degree of voltage compensation achieved is still applied to this case.

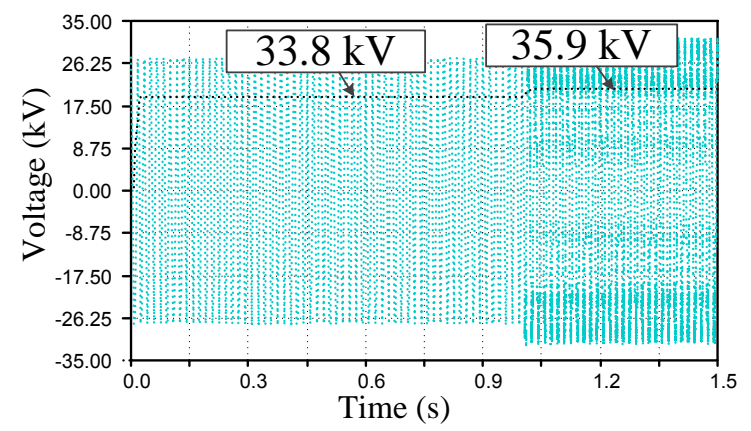

Fig. 11. Voltages for the $34.5 \mathrm{kV}$ Bus for the Amorphous Alloy Twin-Tripler Reactor - before and after the load relief.
Concerning the currents involved in the functioning of the mentioned reactor, these possess the behaviour indicated in Figure 12, which reveal the star up transient behaviour, the condition prior to load relief and the situation immediately after the occurrence of the phenomenon.

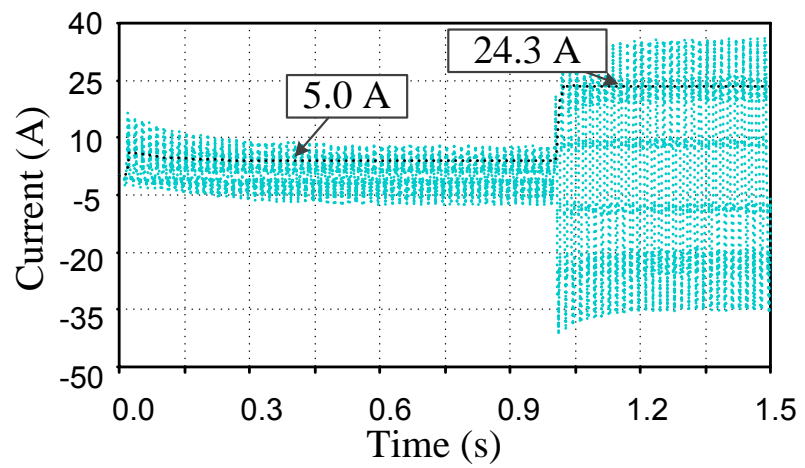

Fig. 12. Currents Absorbed by the Amorphous Alloy Reactor - Before and After the Load Relief.

As can be seen, in the initial condition, the amorphous alloy reactor under study absorbs a current around 55\% when compared to the silicon steel device. This fact is important as being the first element of impact on the reduction of Joule loss. Once the load relief has occurred, this magnitude approximates the same value as that absorbed by the silicon steel device, therefore, the reactor is consuming the same amount of reactive power to the previous device. On the other hand, the magnetic induction value is presented initially as $1.51 \mathrm{~T}$. In accordance with the values provided in Figure 2, the corresponding iron losses are shown in the order of $0.25 \mathrm{~W} / \mathrm{kg}$ (a value of about $10 \%$ of the former). This in itself already shows a strong attraction towards this new reactor concept, due to its expressive reduction in losses, from that made from iron. As to the full saturated operation, the magnetic induction value is around $1.60 \mathrm{~T}$, which leads to iron losses of around $0.37 \mathrm{~W} / \mathrm{Kg}$. This value is approximately equal to $12 \%$ to that of silicon steel.

With the aim of showing the harmonic distortion performances in more details, in Figure 13 the corresponding voltage and current waveforms are presented. In light of these, it is shown that the harmonic orders of greater relevance are the following: 11th order $\left(\mathrm{I}_{11}=3.88 \mathrm{~A}\right)$ and the $23 \mathrm{rd}$ order $\left(\mathrm{I}_{23}=1.36 \mathrm{~A}\right)$, these values were observed during the load relief conditions. The resulting THD for the busbar voltage of $34.5 \mathrm{kV}$, after load rejection, is presented as $4.2 \%\left(\mathrm{~V}_{11}=4.2 \%\right.$ or $874 \mathrm{~V}$ and $\mathrm{V}_{23}=0.36 \%$ or $74.6 \mathrm{~V}$, phase values).

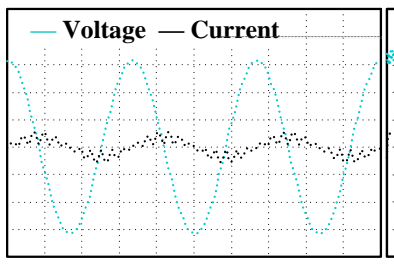

(a)

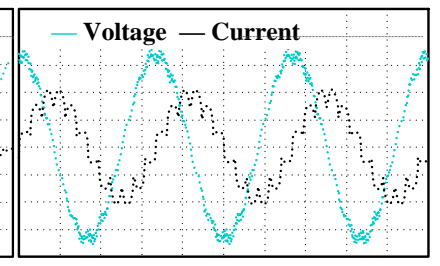

(b) 
Fig. 13. Details of the voltage and current waveforms for the two operative conditions for the Amorphous Alloy Reactor: (a) before the load relief and (b) after the load relief.

\section{Conclusion}

Through a consideration of the most distinct applications for amorphous alloys, it is clear that these in fact have become more competitive in relation to silicon steel, as the development of magnetic material makes the construction of equipment more efficient. In line with this recognition, the results reported herein demonstrate that for applications involving the use of saturated reactors, the employment of amorphous alloys, in substitution of silicon steel, proves to be quite attractive in terms of energy efficiency in the saturated core regulator. In fact, the predictions made when stablishing comparative terms of magnetic flux versus excitation current for these two commercially available materials, show themselves to be consistent with the computational performances obtained.

In relation to the effectiveness of the voltage regulation process, the studies clearly indicate that the employment of reactors built upon amorphous materials are shown to be equally appropriate to the aims established in this paper. Furthermore, when the positive aspects related to the Joule and iron losses are conciliated, gains can be implied for the use of the new material referred to herein.

A point worth noting refers to the fact that the design procedures in practice have shown that the use of amorphous materials requires, for the same power and voltage levels, magnetic cores of larger volume and weight, a fact that partially mitigates the quantitative measures presented for the reduction of iron losses.

Relevant impacts were also considered in the context of harmonic distortions produced by saturated reactors under nominal load conditions. As seen, under these circumstances, the effects are lessened and, under the action of load relief, the results indicate that there were no prejudicial losses.

One aspect that should not be overlooked is directly habituated to the relief of currents under the system's normal operating conditions. As already seen, faced with the fact that the reactors based on amorphous alloys absorb expressively lower currents, this constitutes a relief to the transmission system at exactly the moment of heavier loads. This reduces losses and promotes the liberation in terms of the capacity to transport power by the feeders, as is desired.

\section{Acknowledgement}

The authors express their gratitude to FAPEMIG, CAPES $\mathrm{CNPq}$ for the $\mathrm{PhD}$ and undergraduate research grants directed to the Electrical Engineering Faculty of UFU.

\section{References}

[1] International Standard - IEC 60038. Ed. 6.2, 1997.
[2] J. C. Oliveira, A. B. Vasconcellos, R. Apolônio, "Saturated Core Reactor Static Compensator: Computational versus Experimental Performance Analysis", 11 ${ }^{\circ}$ International Conference on Harmonics and Quality of Power (ICHQP), New York, Sep. 2004.

[3] R. S. Vedam and M. S. Sarma. Power Quality - VAr Compensation in Power Systems, CRC Taylor \& Francis Group, United States of America, 2009.

[4] J. A. F. Barbosa Jr., J. C. Oliveira, F. P. Santilio, I. N. Gondim, A. B. Vasconcellos, J. M. Pacheco, "A Computational ATP Model and Validation to Represent Saturated Core Reactors", 9th IEEE/IAS International Conference on Industry Applications (IX Induscon), São Paulo-SP, Nov. 2010.

[5] L. Mandache and K. Al-Haddad. "High Precision Modeling of Saturable Transformers used as Voltage Regulators", IEEE International Symposium on Industrial Electronics (ISIE), Montréal, Québec, Canada, Jul. 2006.

[6] J. C. Oliveira, et. al. "A Strategy for Voltage Regulation in Eolic Systems", $11^{\text {th }}$ International Conference on Harmonics and Quality of Power (ICHQP), New York, Sep. 2004.

[7] R. Hasegawa and D. Azuma. "Impacts of amorphous metalbased transformers on energy efficiency and environment", Journal of Magnetism and Magnetic Materials, vol. 320, 2008, pp. 2451-2456.

[8] J. A. F. Barbosa Jr. et al. "Modelling and Validation of the Magnetizing Curve to Represent Saturated Core Reactor Using ATP Simulator", International Conference on Renewable Energies and Power Quality - ICREPQ'12, Santiago de Compostela, Spain, 2012.

[9] S. V. Kulkarni, S. A. Khaparde, Transformer Engineering - Design and Practice, Marcel Dekker, Inc, New York, 2004. 